\title{
Survival analysis of A.C. machines in the diamond industry using the Kaplan-Meier estimator
}

\author{
Vladislav A. Shevchuk ${ }^{1}$, Oleg P. Muravlev ${ }^{1}$, and Olga O. Stolyarova ${ }^{1, *}$ \\ ${ }^{1}$ National Research Tomsk Polytechnic University, 634050 Tomsk, Russia
}

\begin{abstract}
The article deals with the survival analysis of a.c. machines operating under the conditions of the underground mine "Mir" (Yakutia). The estimation of their survival is carried out using the Kaplan-Meier estimator. The main advantage of this method is in the opportunity to process censored (incomplete) data, which in turn leads to the accuracy of results. The statistical data used in this article were obtained from maintenance books and reports made by a repair team working in the considered mine. This analysis allows not only identifying faulty electric machines and their fault-free operation but also the faulty components of a certain electric motor.
\end{abstract}

\section{Introduction}

Significant advances have been recently achieved in the field of diagnostics and maintenance of electric machines. It is associated with the improvement of the system of planned preventive maintenance and the introduction of modern high-technology diagnostic techniques and information technologies. However, electric motors operating in the diamond industry can be occasionally exposed to some sudden breakdowns and emergencies. These problems lead to economic losses during the period of the machine down time.

The goal of this article is to carry out the survival analysis of a.c. machines operating in the underground mine "Mir" (Yakutia) using the Kaplan-Meier estimator.

Initially, the methods of survival analysis were widely used in medicine and biology, as well as in economics and social science. Then, they gradually gained in popularity and found their application in mechanical engineering in the form of reliability analysis. However, the methods of survival analysis are still not widespread in Russia [1, 2].

\section{Experimental procedure}

The key feature of survival analysis is the possibility to process censored (incomplete) data, which enables to enhance the data selection and leads to the accuracy increase of results.

\footnotetext{
* Corresponding author: muravlevaoo@tpu.ru
} 
Censored data are observations which do not contain any data about the development of the corresponding event. The survival function for data selection with censored data can be presented as a formula of the Kaplan-Meier estimator [3 - 6]:

$$
S(t)=\prod_{j=1}^{t}\{[n-j] /[n-j+1]\}^{\sigma(j)}
$$

where $n$ is the number of events (failures), $j$ is the order number of a certain event; $\sigma(j)=1$, if the observation is complete and 0 if the observation is censored.

$S(t)$ from (1) plot of function is called a survival curve. Sloping plot indicates high survival; abrupt plot shows low survival. The survival curve construction allows comparing the distribution for two or more groups [4].

The underground mine "Mir" was chosen as the object for analysis, since a major share of electric machines failures has occurred within the period from February 2012 till August 2017. Electric machines ranging from pumps to industrial combines that operate in different mining equipment were chosen for data selection. Figure 1 illustrates the comparison of survival of two most widely used electric machines.

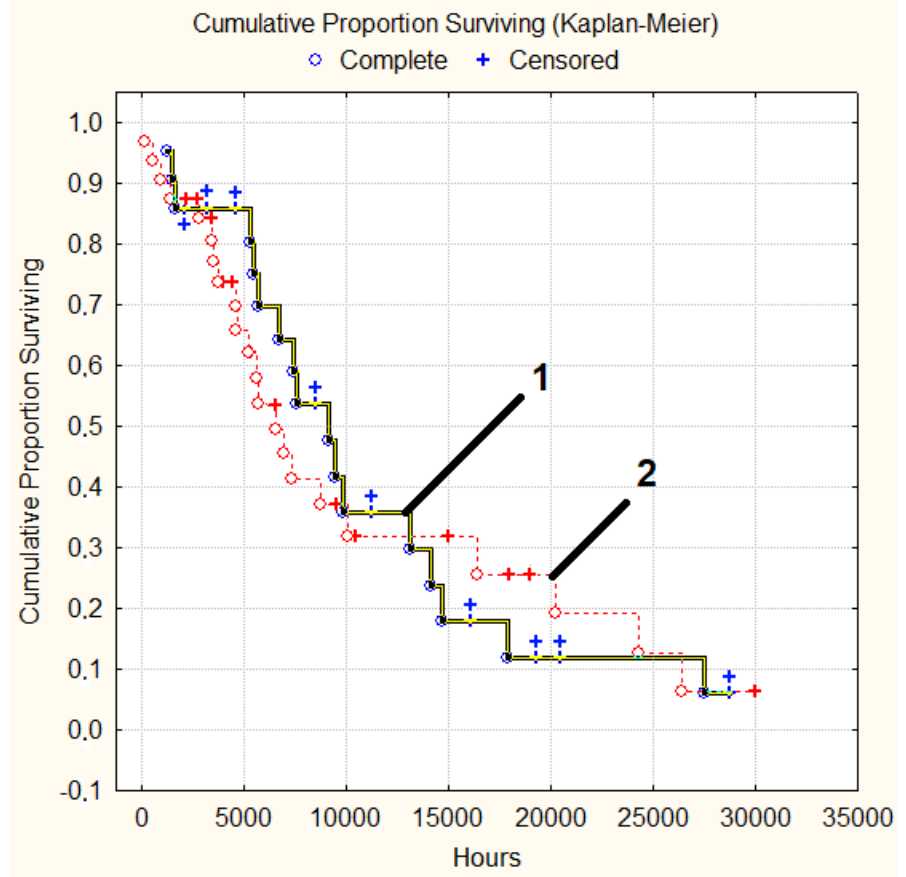

Fig. 1. Comparison of survival of electric machines in the underground mine "Mir".

The diagram shows that for electric machines of d2PRW X250/31-KL model (presented by curve 1) the first 25000 hours of operation are critical. The survival probability abruptly drops to $85 \%$, and the survival probability until 10000 is equal to $35 \%$. Despite the fact that curve 2 for d2PRWX280 model is located more left-sided than curve 1 , it is longer than curve 1 . This indicates that curve 2 has higher survival index. Thus, it can be assumed, that electric model of d2PRW X250/31-KL is more defective. 


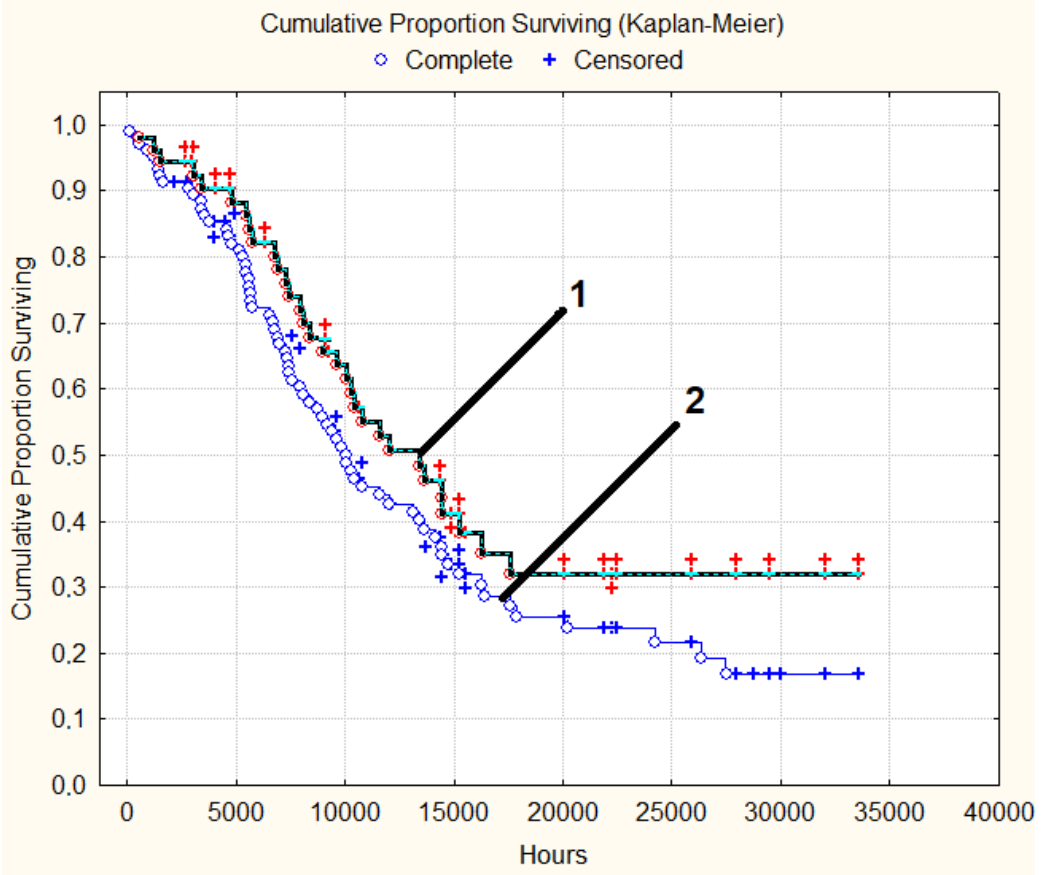

Fig. 2. Comparison of electric machine survival in the underground mine "Mir" with and without an electric motor of d2PRW X250/31-KL model.

Fig. 2 presents the survival plot of an electric machine operating at the mine "Mir" without an electric motor (curve 1) and with an electric motor of d2PRW X250/31-KL model. The rate of survival has considerably increased since the survival probability till 34000 hours is equal to $31 \%$ compared to $18 \%$. Thus, it can be assumed, that in order to increase the survival rate some extra diagnostic and preventive measures are required.

The faultiest component as well as the critical time for each component can be detected using the Kaplan-Meier estimator. To do this we should know some supplementary data about faults. These measures in turn help to plan scheduled preventive maintenance for a particular electric motor [1].

The faultiest components (Fig.3) in our case with the lowest survival rate are the elements of bearing assembly (curve 1). It was defined that by achieving some certain operating time there are some sharp drops of survival curve during the motor operation. The survival probability is $80 \%$ if the operating time equals 5000 hours and $30 \%$ relatively if the operating time is 9000 hours.

There is also necessary to pay attention to the survival curve of shaft components (curve 2). It is obvious from this curve that the main number of failures occurs within the first 5000 hours. The survival probability till 5000 hours is equal to $41 \%$. In accordance to the survival curve of stator components (curve 3 ) the abrupt drop led to $28 \%$ of survival probability till 10000 hours. 


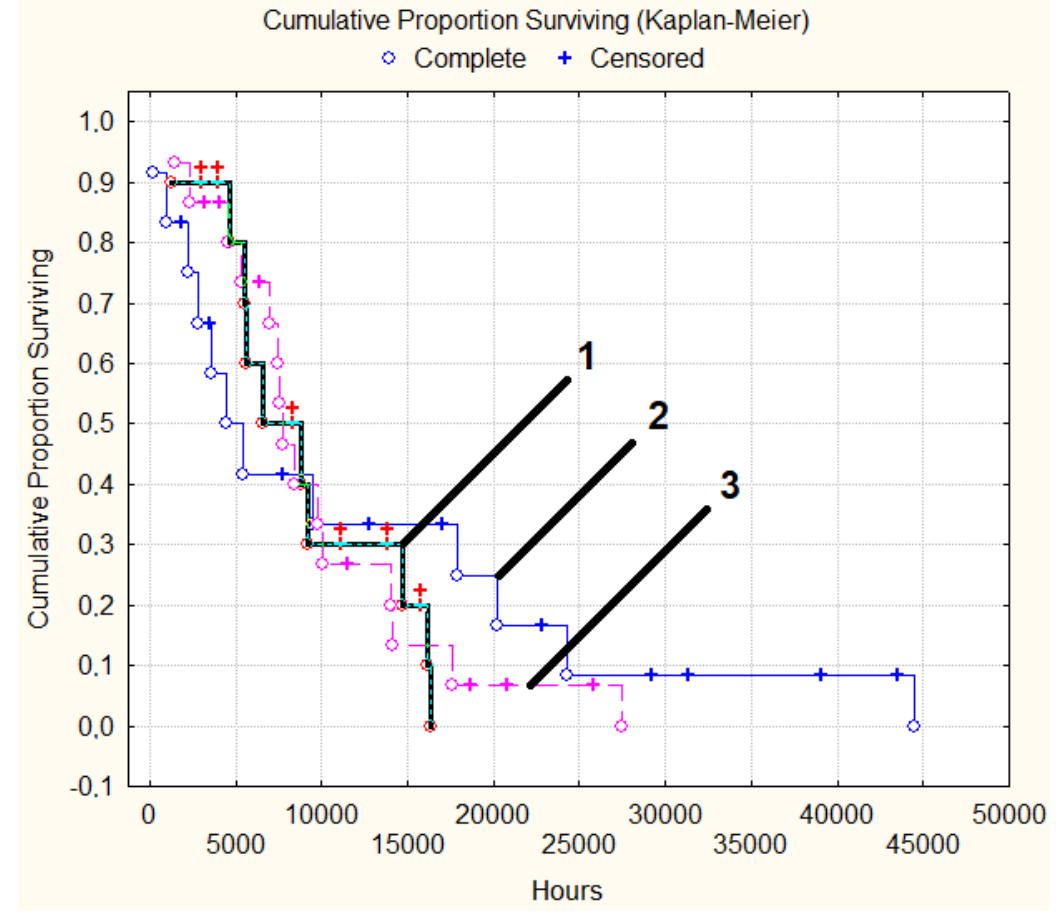

Fig. 3. Comparison of survival for electric motor components of d2PRW X250/31-KL model.

\section{Conclusion}

To sum up, using the Kaplan-Meier estimator it is possible to detect the faultiest model of electric motors in a system as well as the faultiest components in a certain electric motor. Since the survival estimation is calculated with reference to time, some certain faulty components can be defined in advance. The Kaplan-Meier method was recommended for application and adopted by the department of technical diagnostics of Specialized Repair and Construction Administration (Mirny, Yakutia).

\section{References}

1. J. Huang, M. Pflueger, "Electrical Submersible Pump Survival Analysis" (2011)

2. B. Tsybikov, E. Beyerleyn, P. Tyuteva, MATEC Web Conf. 91, 01034 (2017), DOI: 10.1051/matecconf/20179101034

3. J. P. Klein, H. C. van Houwelingen, J. G. Ibrahim, T. H. Scheike, Handbook of survival analysis (CRC Press, 2014), DOI: 10.1002/bimj.201400252

4. A. van den Hout, Multi-state survival models for interval-censored data (CRC Press, 2017)

5. V. Borovikov, STATISTICA. The art of data analysis through a computer (Piter, 2003)

6. V. A. Shevchuk, O. P. Muravlev, O. O. Stolyarova, V. P. Shevchuk, MATEC Web Conf. 91, 01033 (2017), DOI: 10.1051/matecconf/20179101033 